\title{
Research on the Status and Evaluation of Laparoscopic Colorectal Surgery
}

\author{
Ruizhi Hou, Ruizhe Hou, Yan Liu \\ China-Japan Union Hospital of Jilin University, Changchun, Jilin Province, 130021
}

Keywords: Status, Evaluation, Laparoscopic Colorectal Surgery

\begin{abstract}
In order to promote the progress of laparoscopic colorectal surgery in China, this paper introduces the current situation of laparoscopic colorectal surgery at home and abroad, points out the problems in the development of laparoscopic colorectal surgery in China, the mechanism of tumor incision after laparoscopic surgery, measures to reduce the implantation of tumor incision and the promotion of laparoscopic colorectal surgery measures were fully described.
\end{abstract}

\section{Introduction}

Laparoscopic early 90s after successful resection of the colon, the technology slowly into the conventional colon resection, but the development is far slower than other laparoscopic surgery. At present, laparoscopic colon surgery has proved to be a safe operation. Compared with the traditional open colectomy, although the operation time is slightly longer (30-50) minutes, the postoperative recovery, average hospitalization days, complications and mortality are less common than traditional colon resections and the current results are obtained during the continuous improvement of laparoscopic colon surgery. The effectiveness of laparoscopic colon surgery on the margins of the gut segment and lymphadenectomy has been shown to be the same as for open colectomy, demonstrating that laparoscopic techniques are entirely capable of performing oncologic surgery. The results of the initial prospective study showed no significant difference in postoperative survival between the two and in terms of incision implantation as well as earlier reports, comparable to those of the traditional open abdominal resection [1]. Long-term prospective randomized bulk case studies have not yet come out, and this is routine for laparoscopic colon cancer surgery is necessary.

\section{The Tumor Incision Planting Mechanism}

For laparoscopic surgery for colon cancer, many surgeons are understandably cautious without a completed understanding of its efficacy. In particular, since the reports of incision in the abdominal wall have been reported, many clinical and animal experiments have been conducted to find out its causes and preventative measures. At the same time, attention has also been paid to incision planting problems that have occurred in traditional laparotomy. Now that the mechanism of tumor incision caused by laparoscopic surgery is mainly:

(1) Exfoliated tumor cells; (2) incision injury at Trocar and leakage of CO2 along Trocar; (3) surgical equipment, adhesion of tumor cells caused by incision "pollution"; (4) tumor cell aerosolization; (5) different pouch gas pairs The internal environment, including the impact of acid-base balance; (6) artificial pneumoperitoneum on cellular immunity; (7) blood dissemination. If pneumoperitoneum can solve the incision tumor cell planting or extensive peritoneal metastasis, you can further increase the safety of laparoscopic gastrointestinal cancer radical surgery. In order to reduce the incidence of these phenomena, you can try to choose the following measures: (1) after surgery with 5- FU incision; (2) intraperitoneal chemotherapy and intravenous chemotherapy; (3) surgical excision of Trocar incision; (4) clinical use of helium gas Abdominal instead of CO2 pneumoperitoneum; (5) using humidification of the humidification of $\mathrm{CO} 2$ to reduce the atomization of tumor cells, the use of sealed glands to reduce the leakage of tumor cells due to gas and attached to the incision; (6) pneumoperitoneum laparoscopic gastrointestinal Tumor radical surgery. By incision protection and taking appropriate precautions, it is possible to reduce 
laparoscopic incision implantation rates to substantially the same levels as laparotomy.

The application of laparoscopy in colorectal surgery is technically proven to be feasible, that is, in the operation of the card, operation time, complication rate and other traditional open surgery is the same, but the long-term efficacy of the tumor remains to be prospective Randomized large case studies, and prospective randomized comparative study of laparoscopic and open colon resection has been conducted in Europe and the United States, our country in this area is still lack of scientific prospective clinical studies, mostly some clinical reports. Hope that in the Chinese Medical Association branch of laparoscopic surgery and endoscopic surgery under the guidance of multi-center prospective clinical research cooperation in order to clarify the status of laparoscopic surgery in colorectal surgery [2].

Laparoscopic technology in the late 1980s in the rise around the world, in the early 90s in various surgical fields have been widely carried out in the mid-90s started in gastrointestinal surgery has been widely carried out because of the application of ultrasonic scalpel and surgical techniques continue Improve, laparoscopy in colorectal surgery has made considerable progress in the application of the new century, laparoscopic technology and surgical fields with various professional, laparoscopic technology will become an important colorectal surgical treatment.

\section{The Evaluation of Laparoscopic Colorectal Surgery Efficacy and Safety}

And laparoscopic cholecystectomy, laparoscopic assisted colon surgery in our country compared with the start not too late abroad, but the difference is that laparoscopic cholecystectomy after more than 10 years of development, both in terms of the number and quality of surgery in our country The world advanced level, and laparoscopic colorectal surgery because of economic constraints and people's concept of constraints, regardless of the number of operations, types, time, quality is still a gap, and now the number of small surgery, surgery a long time, more than small and medium hospitals Large-scale teaching hospitals, non-colorectal surgery professionals who are not proficient in traditional colorectal surgery, the lack of prospective case-control studies, surgical indications and non-standard operating techniques [3]. It is recognized that the learning curve of laparoscopic colorectal surgery is about 50 cases, while our country is generally still in the learning stage, which is detrimental to the development of laparoscopic colorectal surgery in our country.

As with laparoscopic cholecystectomy, laparoscopic assisted colorectal surgery in our country compared with foreign start not too late, but the difference is that laparoscopic cholecystectomy after more than ten years of development, both in our current number and quality of surgery ranking the world's advanced level. Due to the restriction of economic conditions and the restriction of people's concepts, laparoscopic colorectal surgery has a lot of gaps in terms of quantity, type, time and quality of operation. Laparoscopic colorectal surgery is mainly affected by the level of economic development, the concept of surgical techniques and other effects, manifested in the small number of surgery, surgery for a long time, small hospitals more than large teaching hospitals, non-colorectal surgery surgeon, the traditional colorectal surgery Not proficient, the lack of prospective randomized controlled case studies, surgical indications and technical non-standard operation. Now recognized as a laparoscopic colorectal surgery learning curve of about 50 cases, while our country is still generally in the learning stage, which for the development of laparoscopic colorectal surgery in China is very unfavorable. For laparoscopic surgery for colon cancer, it is understandable that many surgeons are cautious without full knowledge of their efficacy. In particular, since the reports of abdominal incision implantation, many clinical and animal experiments have been conducted to clarify its causes and preventative measures, while noting the problem of incision in traditional laparotomy [4]. Now that the mechanism of tumor incision implantation after laparoscopic surgery are mainly: (1) the direct implantation of tumor cells off; (2) Tro-car incision injury and CO2 along the Trocar leakage; (3) surgical procedures, the instrument attached to tumor cells caused by incision "Pollution"; (4) aerosolization of tumor cells; (5) different inflation gases on the body's internal environment, including acid-base balance; (6) artificial pneumoperitoneum on cellular immunity; (7) blood dissemination. If pneumoperitoneum can solve the incision tumor cell planting or extensive peritoneal metastasis, you can further 
increase the safety of laparoscopic gastrointestinal cancer radical surgery. To reduce the incidence of these phenomena, you can try to use the following measures: (1) after surgery with 5- FU incision; (2) intraperitoneal chemotherapy and intravenous chemotherapy; (3) surgery after removal of the incision in the Tro-car; (4) clinical use of helium Pneumoperitoneum instead of CO2 pneumoperitoneum; (5) using humidification of the humidification of $\mathrm{CO} 2$ to reduce the atomization of tumor cells, the use of sealed glands to reduce the leakage of tumor cells due to gas and attached to the incision; (6) pneumoperitoneum laparoscopic stomach Gastrectomy. By incision protection and taking appropriate precautions, it is possible to reduce laparoscopic incision implantation rates to substantially the same levels as laparotomy.

Laparoscopic colorectal surgery largely depends on the surgeon, so it is recommended that laparoscopic colorectal cancer resection be initiated only after at least 20 laparoscopic benign colon tumor resections. The initial choice of simple, easier surgery, such as right hemicolectomy, and surgery under the guidance of an experienced surgeon; follow the principle of radical tumor surgery, that is, without contact with the tumor prior to treatment of blood vessels, adequate margin, complete Lymphadenectomy; take precautions to avoid tumor cell proliferation and abdominal wall planting.

\section{Chinese Clinical New Technology and Health Economics Evaluation}

In our country, there is still a lack of scientific prospective clinical research, mostly some retrospective clinical reports and the intensity of argumentation is not high. Hope that in the Chinese Medical Association Branch of laparoscopic surgery and endoscopic surgery under the guidance of multi-center prospective clinical research cooperation in order to clarify the status of laparoscopic surgery in colorectal surgery. The good news is that the provinces and cities across the country's minimally invasive surgical team is constantly established and actively carry out activities, and some have developed a standardized standard for laparoscopic colorectal surgery. Chinese large population, lack of resources, minimally invasive colorectal surgery for health economics evaluation is particularly necessary, which is deciding whether to promote the technology under the current conditions has an important guiding significance. Cost-effectiveness analysis for laparoscopic and open colorectal surgery in foreign countries already have articles in this area, and our country has not yet had this research, with the continuous development of minimally invasive technology and social health insurance system in the full implementation of our country, we need to laparoscopic colorectal surgery health economics evaluation, and continuously improve the efficacy and surgical techniques, shorten the operation time, reduce complications and reduce the cost of surgery for the benefit of the majority of patients [5]. Laparoscopic technology in the late 1980s in the world rise in the early 90s in various surgical fields are widely carried out in the mid 90s began in gastrointestinal surgery has been widely carried out because of the ultrasonic knife, all kinds of cutting stapler application And surgical techniques continue to improve, the application of laparoscopy in colorectal surgery has made great strides, the new century, laparoscopy and surgical fields will be combined with various professions, laparoscopy will surely become an important treatment of colorectal surgery means.

\section{Conclusions}

In the late 1980s, laparoscopic techniques started to rise around the world and were widely used in various surgical fields in the early 1990s. Since the mid-1990s, gastrointestinal surgery has been widely carried out. Due to the application of ultrasonic knives and cutting staplers Advances in surgical techniques and advances in the use of laparoscopy in colorectal surgery have made great strides. In the new century, laparoscopic techniques and various fields of surgery are combined. Laparoscopic surgery will surely become an important treatment for colorectal surgery means. 


\section{References}

[1] Wang Cunchuan, Xu Yirhao, Chen X. Laparoscopic colorectal surgery in 55 cases [J]. Chinese Journal of Surgery. 2000 (04) 42-45.

[2] Liu Guoli. Chinese status of laparoscopic surgery [J]. Chinese Journal of Surgery. 1999(10) 62-67.

[3] Yu Baoming. Evaluation of laparoscopic colorectal surgery [J]. Surgical Theory and Practice. 1999 (03) 50-58.

[4] Wang Xianhua, Li Mingzhen, wisdom, Xie Xi, Chen Rongfei. Laparoscopic assisted resection of colorectal cancer in 15 cases [J]. Chinese Journal of general surgery. 1999 (04) 50-78.

[5] Zheng Minhua, Wang Hao, Yu Baoming. Laparoscopic colorectal surgery [J]. Chinese Journal of Practical Surgery. 1998 (05) 62-77. 